\title{
Immunological corollary of the pulmonary mycobiome in bronchiectasis: the CAMEB study
}

\author{
Micheál Mac Aogáin (101, Ravishankar Chandrasekaran ${ }^{1,11}$ \\ Albert Yick Hou Lim², Teck Boon Low ${ }^{3}$, Gan Liang Tan ${ }^{4}$, Tidi Hassan ${ }^{5}$, \\ Thun How Ong ${ }^{4}$, Amanda Hui Qi Ng ${ }^{6}$, Denis Bertrand ${ }^{6}$, Jia Yu Koh ${ }^{6}$, \\ Sze Lei Pang ${ }^{7,8}$, Zi Yang Lee ${ }^{7}$, Xiao Wei Gwee ${ }^{7}$, Christopher Martinus ${ }^{7}$, \\ Yang Yie Sio ${ }^{7}$, Sri Anusha Matta ${ }^{7}$, Fook Tim Chew ${ }^{7}$, Holly R. Keir ${ }^{9}$, \\ John E. Connolly ${ }^{10}$, John Arputhan Abisheganaden ${ }^{2}$, Mariko Siyue Koh ${ }^{4}$, \\ Niranjan Nagarajan' ${ }^{6}$, James D. Chalmers ${ }^{9}$ and Sanjay H. Chotirmall (1) ${ }^{1}$
}

Affiliations: ${ }^{1}$ Lee Kong Chian School of Medicine, Nanyang Technological University, Singapore. ${ }^{2}$ Dept of Respiratory and Critical Care Medicine, Tan Tock Seng Hospital, Singapore. ${ }^{3}$ Dept of Respiratory and Critical Care Medicine, Changi General Hospital, Singapore. ${ }^{4}$ Dept of Respiratory and Critical Care Medicine, Singapore General Hospital, Singapore. ${ }^{5}$ Universiti Kebangsaan Malaysia, Kuala Lumpur, Malaysia. ${ }^{6}$ Genome Institute of Singapore, A*STAR, Singapore. ${ }^{7}$ Dept of Biological Sciences, National University of Singapore, Singapore. ${ }^{8}$ Institute of Systems Biology, Universiti Kebangsaan Malaysia, Bangi, Malaysia. ${ }^{9}$ Ninewells Hospital and Medical School, University of Dundee, Dundee, UK. ${ }^{10}$ Institute of Molecular and Cell Biology, A*STAR, Singapore. ${ }^{11}$ These two authors contributed equally to this work.

Correspondence: Sanjay H. Chotirmall, Lee Kong Chian School of Medicine, Nanyang Technological University, Level 12 Clinical Sciences Building, 11 Mandalay Road, Singapore 308232. E-mail: schotirmallantu.edu.sg

@ERSpublications

The airway mycobiome in bronchiectasis is associated with clinically significant disease http://ow.ly/MCKj30knVrn

Cite this article as: Mac Aogáin M, Chandrasekaran R, Lim AYH, et al. Immunological corollary of the pulmonary mycobiome in bronchiectasis: the CAMEB study. Eur Respir J 2018; 52: 1800766 [https://doi. org/10.1183/13993003.00766-2018].

ABSTRACT Understanding the composition and clinical importance of the fungal mycobiome was recently identified as a key topic in a "research priorities" consensus statement for bronchiectasis.

Patients were recruited as part of the CAMEB study: an international multicentre cross-sectional Cohort of Asian and Matched European Bronchiectasis patients. The mycobiome was determined in 238 patients by targeted amplicon shotgun sequencing of the 18S-28S rRNA internally transcribed spacer regions ITS1 and ITS2. Specific quantitative PCR for detection of and conidial quantification for a range of airway Aspergillus species was performed. Sputum galactomannan, Aspergillus specific IgE, IgG and TARC (thymus and activation regulated chemokine) levels were measured systemically and associated to clinical outcomes.

The bronchiectasis mycobiome is distinct and characterised by specific fungal genera, including Aspergillus, Cryptococcus and Clavispora. Aspergillus fumigatus (in Singapore/Kuala Lumpur) and Aspergillus terreus (in Dundee) dominated profiles, the latter associating with exacerbations. High frequencies of Aspergillus-associated disease including sensitisation and allergic bronchopulmonary aspergillosis were detected. Each revealed distinct mycobiome profiles, and associated with more severe disease, poorer pulmonary function and increased exacerbations.

The pulmonary mycobiome is of clinical relevance in bronchiectasis. Screening for Aspergillus-associated disease should be considered even in apparently stable patients.

This article has supplementary material available from erj.ersjournals.com

Received: Jan 162018 | Accepted after revision: May 262018

Copyright $\odot$ ERS 2018. This article is open access and distributed under the terms of the Creative Commons Attribution Non-Commercial Licence 4.0. 


\section{Introduction}

Bronchiectasis is a chronic respiratory disease characterised by progressive bronchial dilatation. To date, no therapy has been licensed for its treatment. Geographic regions illustrate preponderance for particular aetiologies, e.g. post-tuberculous disease in countries of endemic infection [1]. Incipient infection in bronchiectasis incites deleterious host inflammatory responses and disease progression. A "vicious cycle" of impaired mucociliary clearance, recurrent infection and chronic inflammation, with established links to bacteria, leads to progressive disease; however, the role of fungi is poorly understood.

The pulmonary microbiome and its association with chronic respiratory disease is an emerging area of research. Culture-independent airway sequencing has revealed novel associations between the airway microbiome and lung disease [2]. To date, most airway microbiome studies have a bacterial focus, an approach that provides disease insight into pathogenesis and prognosis [3, 4]. In contrast, however, sequencing of the fungal microbiome (the mycobiome) has lagged behind and is applied in relatively few studies, and to the best of our knowledge, none in bronchiectasis [5].

Fungal spores are environmentally ubiquitous and hence their inhalation is an inevitable consequence of breathing [6]. While prompt mucociliary and phagocytic clearance occurs in the healthy lung, anatomically abnormal and immunocompromised airways such as in bronchiectasis are at higher risk of fungal acquisition, colonisation and potential disease [6]. Our group and others have performed extensive work characterising fungi in cystic fibrosis (CF)-related bronchiectasis where they colonise and act as pathogens associated with poorer clinical outcome [7-10].

Fungi, particularly Aspergillus spp., cause a range of pulmonary consequences, including allergic, chronic and/or invasive disease [11, 12]. An overzealous host response to Aspergillus, for instance, is clinically important in severe asthma with fungal sensitisation, chronic obstructive pulmonary disease (COPD) and CF [13-15]. The role of fungi in bronchiectasis not due to CF is, however, less well defined. Aspergillus sensitisation and/or allergic bronchopulmonary aspergillosis (ABPA) is a cause $(\sim 10 \%)$ and, importantly, a consequence of bronchiectasis $[6,10,16]$. The role of the mycobiome in bronchiectasis is yet to be comprehensively investigated, and its importance and relevance is clearly highlighted as a priority research area in this field [17].

We characterise for the first time the mycobiome in bronchiectasis, assessing its clinical relevance in two geographically distinct cohorts from the CAMEB study, encompassing four Asian sites across Singapore/ Kuala Lumpur and a single European site in Dundee.

\section{Materials and methods \\ Study population}

Patients with stable bronchiectasis were recruited across three countries as part of the CAMEB study, a cross-sectional Cohort of Asian and Matched European Bronchiectasis. Recruitment included three sites in Singapore (Singapore General Hospital, Changi General Hospital and Tan Tock Seng Hospital (n=124)), one Malaysian site (UKM Medical Centre, Kuala Lumpur $(\mathrm{n}=14)$ ), and an age-, sex- and disease severity-matched group from a single European site (Ninewells Hospital, Dundee, UK $(n=100)$ ), and was conducted between March 2016 and July 2017. Full details on patient inclusion and exclusion criteria, nondiseased controls, and the CAMEB population are provided in the supplementary material. Clinical characteristics, bronchiectasis aetiology and patient demographics are shown in table 1 . The study was approved by the institutional review boards of all participating institutes and all patients gave written informed consent to participate. Further details are given in the supplementary material.

\section{Methods}

Full details on clinical data and specimen collection, statistical analysis, and molecular methods, including mycobiome analyses, sputum quantitative PCR (qPCR) detection of fungi and immunological bioassays, are provided in the supplementary material.

\section{Results}

The pulmonary mycobiome in bronchiectasis is distinct

Culture-independent analysis of the pulmonary mycobiome in bronchiectasis reveals that Ascomycota dominate the airway at the phylum level $(\mathrm{p}<0.01$, Mann-Whitney U-test) (figure 1$)$. Fungal diversity was significantly lower in bronchiectasis, as measured by the number of identified taxa $(p<0.01)$ and the Shannon diversity index $(\mathrm{p}<0.05$, Mann-Whitney U-test) (figure 1$)$. Limitations of fungal internally transcribed spacer (ITS) databases may account for the lower classification percentages observed (95.3\%) compared with bacteria, where genus-level operational taxonomic unit classification of $299 \%$ can be achieved [19]. Despite Ascomycota dominating overall mycobiome profiles, a significant number of bronchiectasis patients $(49 \%(n=116))$ harboured some degree of airway Basidiomycota. 10\% $(n=23)$ had 
TABLE 1 Demographics of the study population including nondiseased (healthy) controls and stable bronchiectasis patients comprising Asian and European matched cohorts

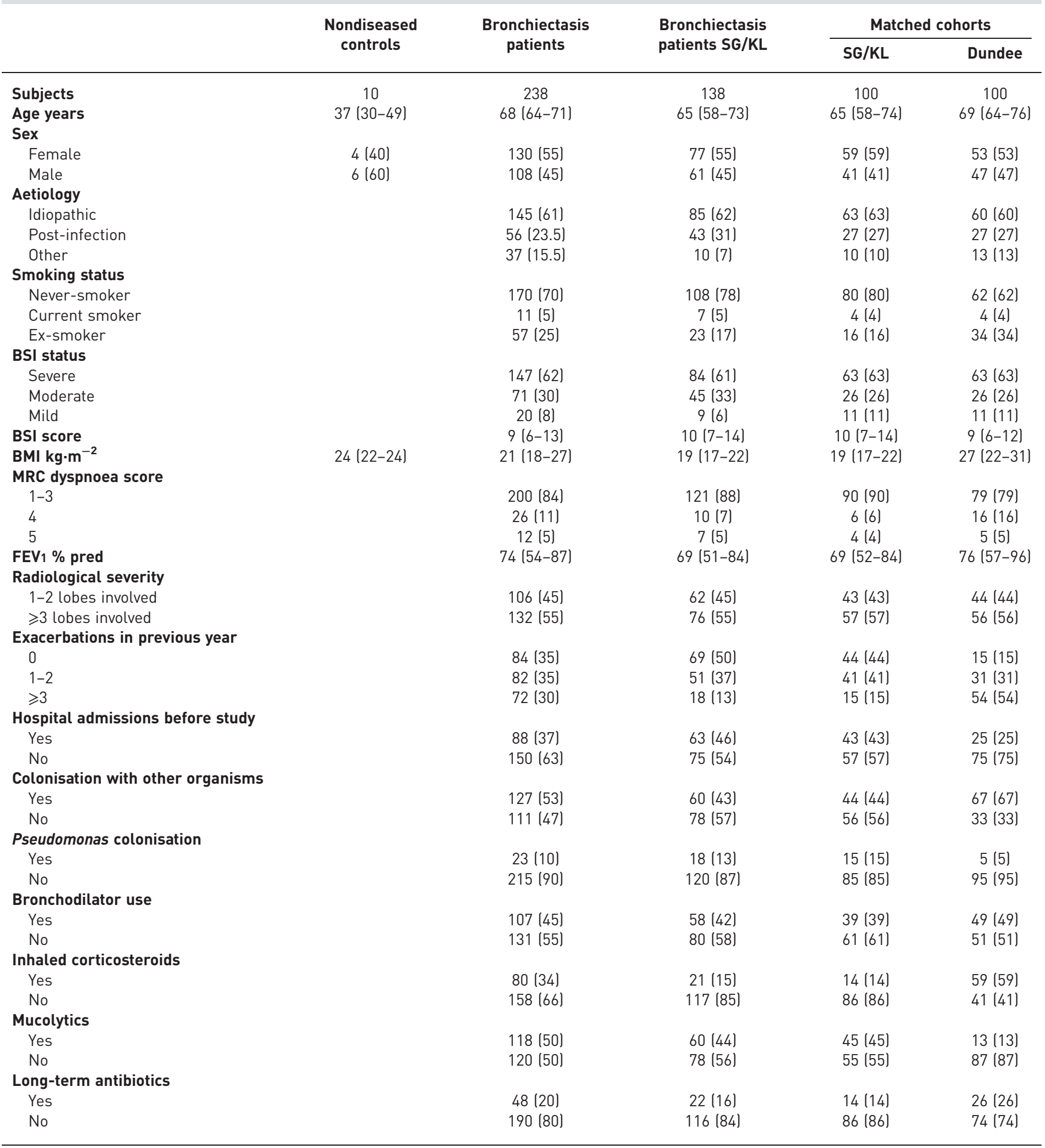

Data are presented as n, median (interquartile range) or n (\%). SG/KL: Singapore/Kuala Lumpur; BSI: Bronchiectasis Severity Index; BMI: body mass index; MRC: Medical Research Council; FEV1: forced expiratory volume in $1 \mathrm{~s}$. Patients in the matched cohorts were matched on age, sex and disease severity according to the BSI. The variables defining the composite BSI score, including BMI, shortness of breath (MRC) dyspnoea score, FEV1 \% pred, radiological severity, number of exacerbations in the preceding year defined by established consensus [18], hospitalisations in the preceding year, microbial colonisation with other organisms and colonisation by Pseudomonas aeruginosa, are also reported. 


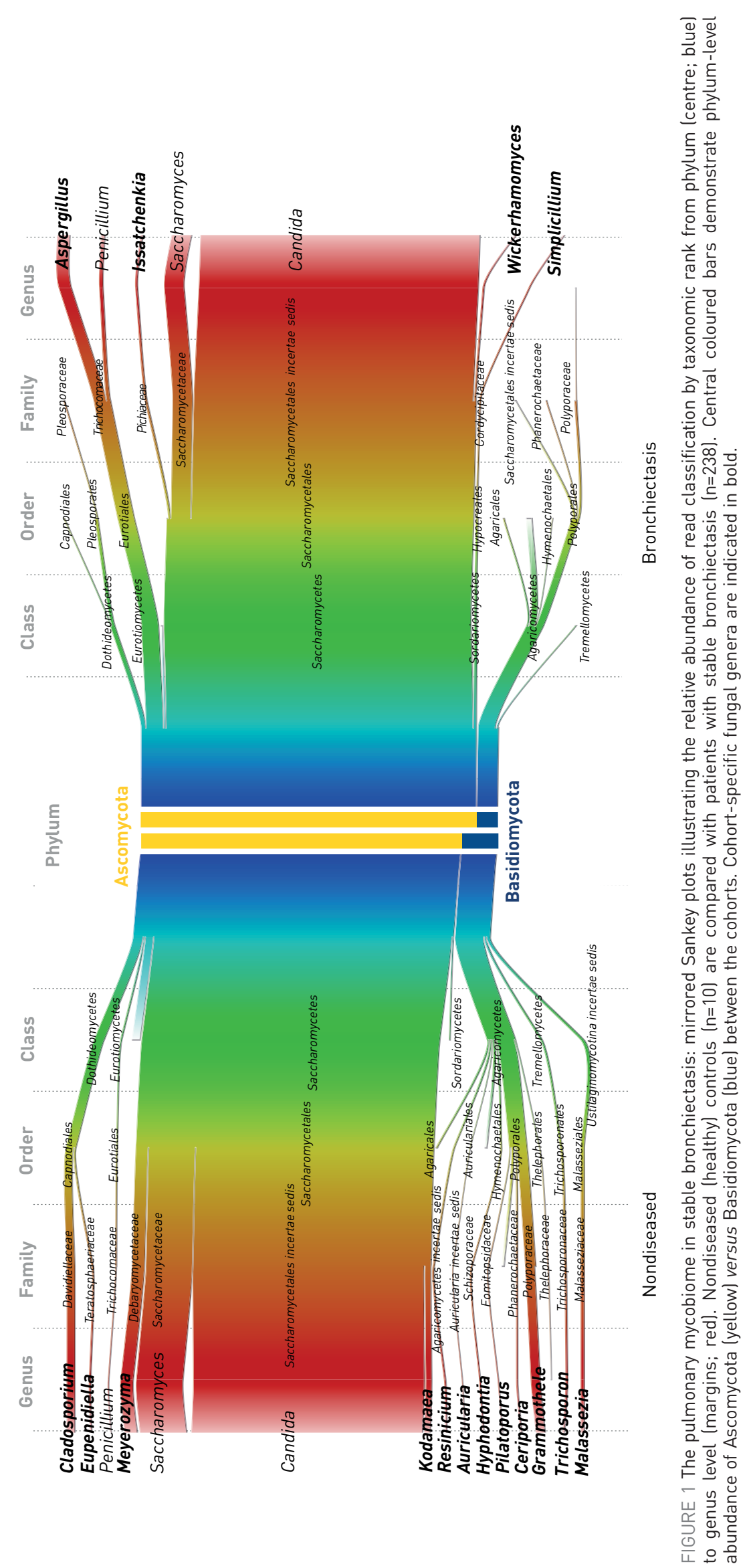


Basidiomycota making up at least a quarter of their overall profile, while $4 \%(\mathrm{n}=9)$ exhibited Basidiomycota-dominant profiles (figure 2a). Candida, Saccharomyces and Penicillium were the most frequently detected genera in both groups, while differentially abundant, bronchiectasis-associated genera included Aspergillus, Cryptococcus, Clavispora, Botrytis and Alternaria (figures 2b and 3a).

In the design of the CAMEB study, $\mathrm{n}=100$ Asian patients (Singapore/Kuala Lumpur) were matched individually to patients from a European cohort (Dundee) by age, sex and total Bronchiectasis Severity Index (BSI) score (table 1). This allowed assessment of findings in two geographically distinct cohorts as well as providing insight into potential regional differences in the bronchiectasis mycobiome. Geographic variation in mycobiome signature was detected (figure 3a). Patients from Singapore/Kuala Lumpur exhibited significant and higher average relative abundances of Simplicillium, Trichosporon and Aspergillus, while patients from Dundee were distinguished by higher abundances of Wickerhamomyces, Clavispora and Cryptococcus $(\mathrm{p}<0.05)$ following assessment of group differences using metastats statistical analysis [20]. Candida, by far the most frequently observed fungal genera, was observed across both cohorts at equal frequency, while patients from Dundee exhibited higher Saccharomyces, Penicillium, Cryptococcus,

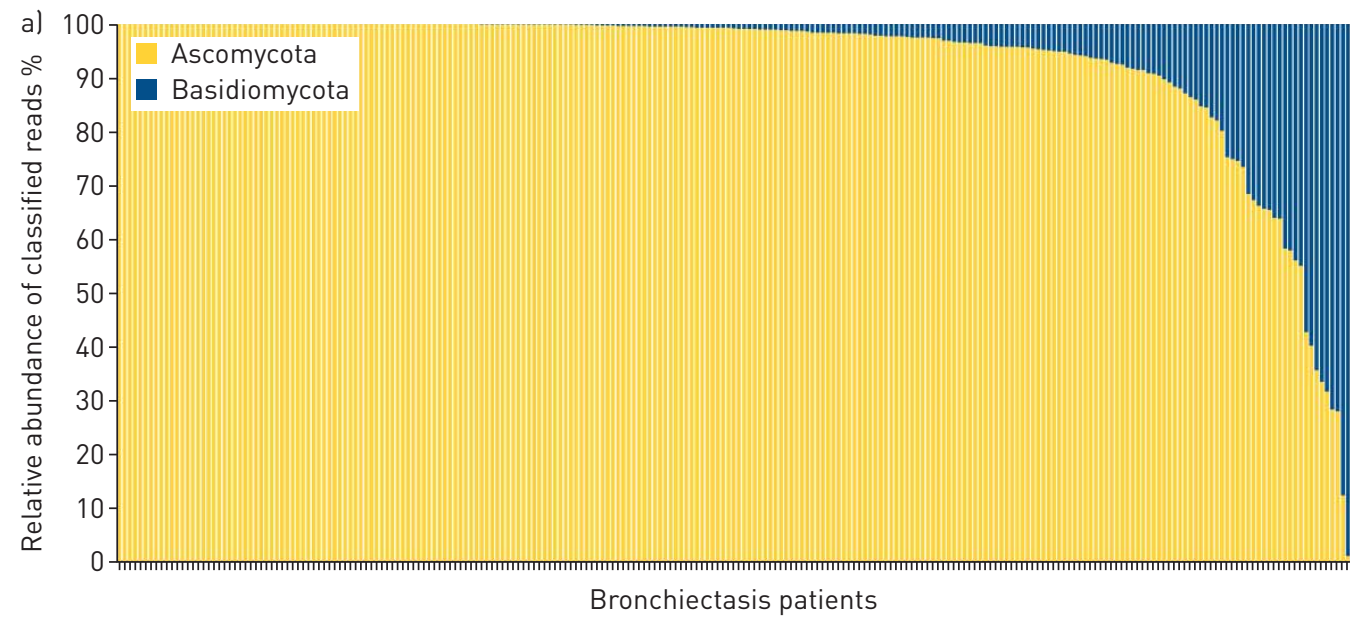

b)

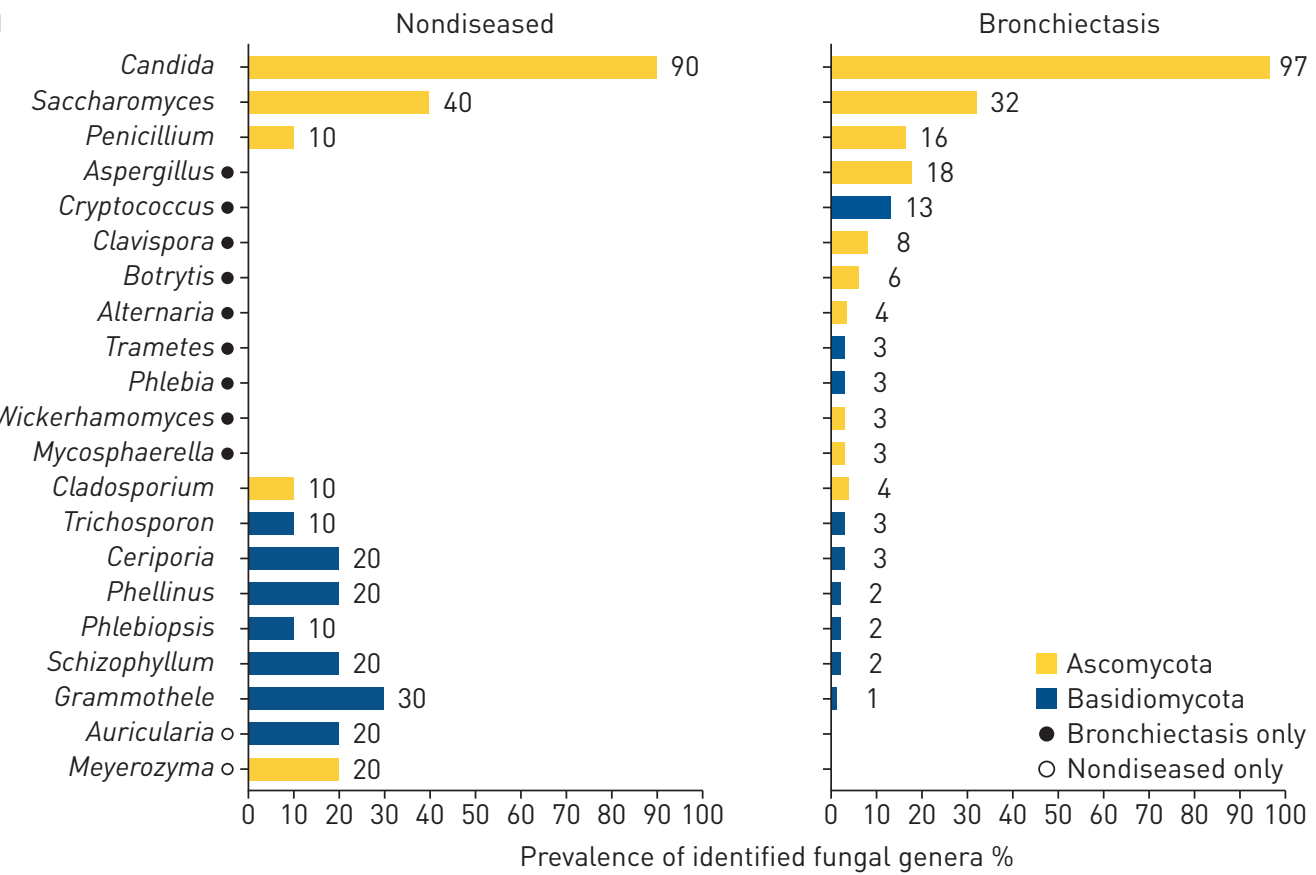

FIGURE 2 The pulmonary mycobiome in stable bronchiectasis: a) individual patient phylum-level classification of the pulmonary mycobiome in stable bronchiectasis $(n=238)$ illustrating the relative abundance of Ascomycota versus Basidiomycota for individual patients, and b) percent prevalence of the top fungal genera (present at $>1 \%$ relative abundance) in nondiseased (healthy) controls $(n=10)$ and patients with stable bronchiectasis $(n=238)$. 
a)

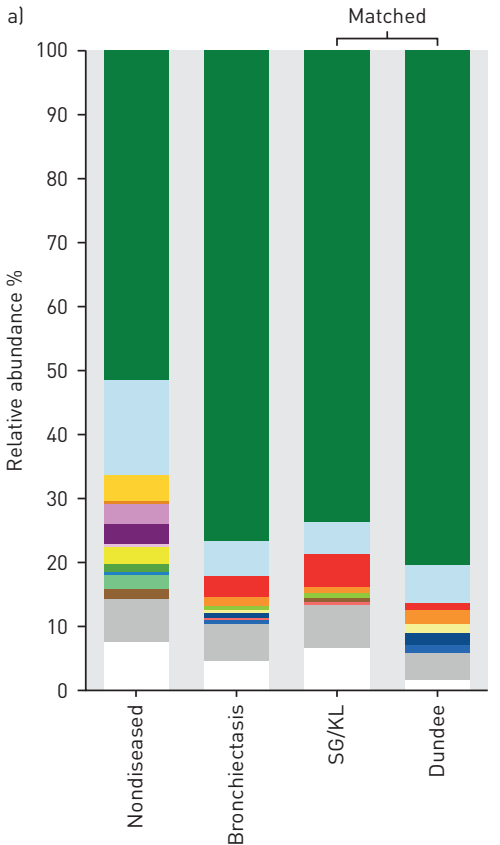

b)

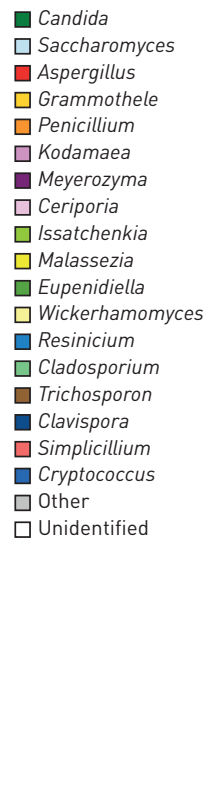

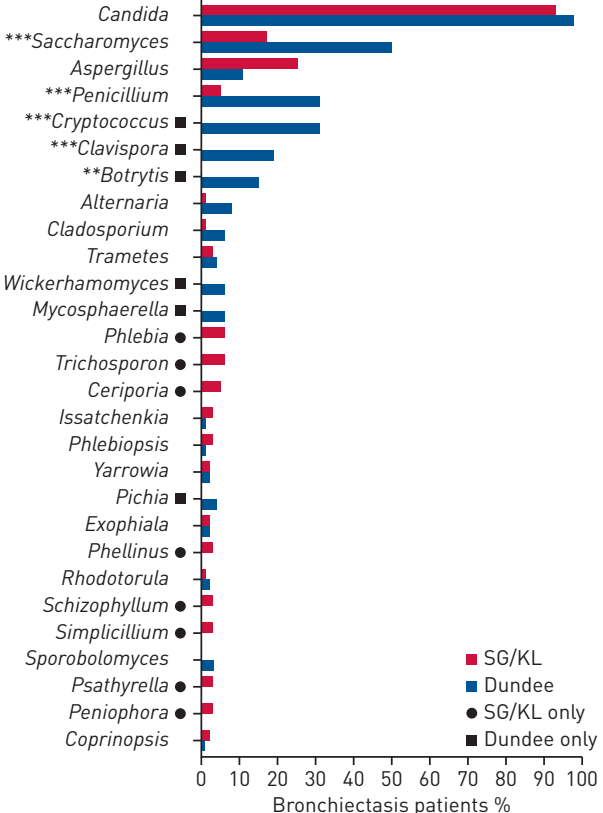

FIGURE 3 The pulmonary mycobiome differs between Singapore/Kuala Lumpur (SG/KL) and Dundee cohorts of stable bronchiectasis. a) Genus-level classification of the pulmonary mycobiome in nondiseased ( $\mathrm{n}=10$ ), stable bronchiectasis $(n=238)$, and matched SG/KL $(n=100)$ and Dundee $(n=100)$ cohorts. The relative abundance of identified taxa is colour-coded. b) Percent prevalence of observed fungal genera (present at $>1 \%$ relative abundance) in matched bronchiectasis cohorts from $\mathrm{SG} / \mathrm{KL}(\mathrm{n}=100$ ) and Dundee ( $\mathrm{n}=100$ ). Significant differences in prevalence between cohorts are indicated. ${ }^{* *}: p<0.01$; $^{* * *}: p<0.001$.

Clavispora and Botrytis in particular. The only fungal genus in the top 10 with higher prevalence in Singapore/Kuala Lumpur was Aspergillus, although this was detected at all the sampled sites (figure $3 \mathrm{~b}$ ). Given our identification of Aspergillus as an exclusive bronchiectasis-associated fungal genus with high airway abundance and its established pathogenic role in other chronic respiratory diseases $[10,12,13,15]$, we pursued it for further investigation.

\section{Aspergillus fumigatus and Aspergillus terreus are identified in bronchiectasis and associate with exacerbations}

We next further characterised the presence of specific Aspergillus species in the airway by qPCR using established protocols published by our group and others [7, 21]. We assessed four major Aspergillus species (A. fumigatus, A. terreus, A. flavus and A. niger) and found that nondiseased individuals had no detectable airway Aspergillus, in agreement with our mycobiome analysis (figure 4a). In contrast, high proportions of bronchiectasis patients (from all sites) had detectable A. fumigatus and/or A. terreus, with no patient demonstrating $A$. flavus or $A$. niger (figure 4a). Interestingly, similar proportions of those recruited from an Asian site had either A. fumigatus and/or A. terreus, while patients from Dundee demonstrated higher $A$. terreus (figure $4 \mathrm{a}$ ), which associates with increased exacerbations (figure $4 \mathrm{~b}$ ). Importantly, significant numbers of patients $(40 \%(n=96))$ harboured both species concurrently. Of these, an equal distribution between groups recruited from Singapore/Kuala Lumpur and Dundee was observed ( $44 \%$ versus 52\%; $\mathrm{p}=0.22$ ) (figure $4 \mathrm{c}$ ). Patients from Singapore/Kuala Lumpur were equally likely to have none, either or both species in their airway, in contrast to patients from Dundee who exhibited a higher likelihood for A. terreus (figure 4c). More exacerbations were seen in patients with A. terreus alone or where both species were present compared with those without any fungi or A. fumigatus alone (figure $4 \mathrm{~d}$ ).

\section{Quantification of A. fumigatus to A. terreus conidial burden ratio and associated exacerbations}

We next quantified $A$. fumigatus and $A$. terreus conidial burden using established protocols published by our group [7]. Based on the number of conidia per gram of sputum, we then classified the detected loads as low $\left(<500\right.$ conidia.g ${ }^{-1}$ sputum $)$, intermediate $\left(500-2000\right.$ conidia $\cdot g^{-1}$ sputum $)$ or high $\left(>2000 \mathrm{conidia} \cdot \mathrm{g}^{-1}\right.$ sputum). Measured conidial burden varied among Aspergillus-positive patients, and was comparable between patients recruited from Singapore/Kuala Lumpur and Dundee (figure 5a). Interestingly, where patients harboured both species concurrently, their proportionality varied: patients from sites in 

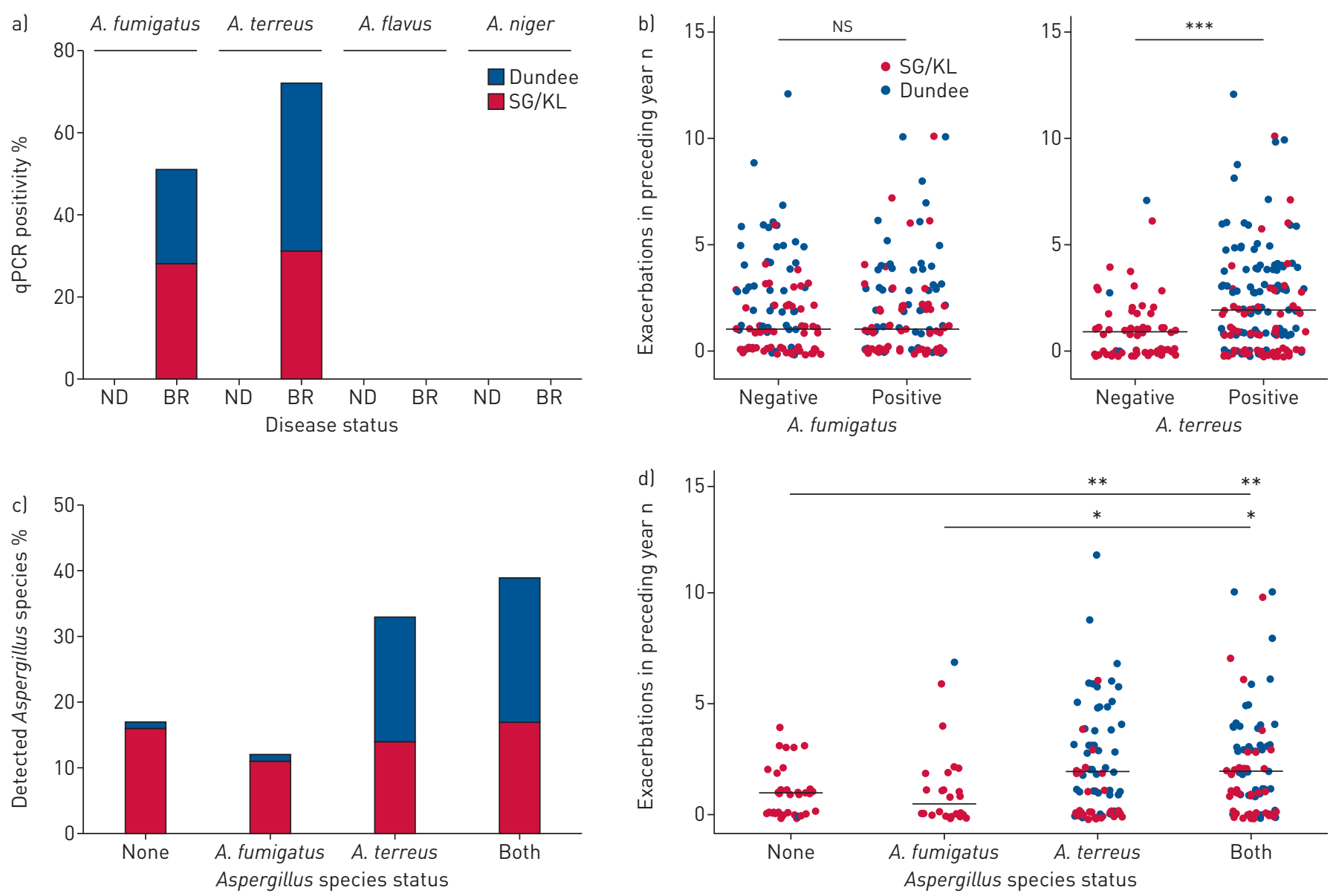

FIGURE 4 Identification of specific airway Aspergillus species and their association with exacerbations in bronchiectasis. qPCR: quantitative PCR; SG/KL: Singapore/Kuala Lumpur; ND: nondiseased; BR: bronchiectasis. al qPCR-based screening for the presence of the major specific Aspergillus species (A. fumigatus, A. terreus, A. flavus and A. niger) in ND (healthy) controls $(n=10)$ and stable $B R(n=238)$. b) Exacerbations for the year preceding study recruitment in patients with qPCR-detectable $A$. fumigatus (left) and $A$. terreus (right). c) Classification of qPCR-detectable Aspergillus species by percentage of the total bronchiectasis patient cohort $(\mathrm{n}=238$ ). Patients are classified as having no detectable species ( $\mathrm{n}=39$ ), A. fumigatus only $(n=26), A$. terreus only $(n=78)$ or both $A$. fumigatus and $A$. terreus $(n=95)$. d) Exacerbations for the year preceding study recruitment in patients with no detectable species, $A$. fumigatus only, $A$. terreus only or both $A$. fumigatus and $A$. terreus. Median number of exacerbations per group is indicated. NS: nonsignificant; ${ }^{*}: p<0.05 ;{ }^{* *}: p<0.01 ;{ }^{* * *}: p<0.001$.

Singapore/Kuala Lumpur exhibited higher proportions of A. fumigatus (median 69\% of conidial load), while the Dundee cohort had a higher A. terreus burden (median $89 \%$ of total conidial load) (figure $5 b$ ). This agrees with our earlier observation of regional variation, where cohorts in Singapore/Kuala Lumpur tended toward more A. fumigatus and the Dundee cohort greater A. terreus either when the fungi exist alone or together. When patients were grouped by detectable Aspergillus species and accompanying conidial burden (using high load as cut-off), a significant association existed between high concurrent conidial burdens of both Aspergillus species and greater exacerbations (figure 5c). Specifically, however, when a high conidial burden of $A$. terreus was identified, either alone or in combination with A. fumigatus, significantly more exacerbations also occurred (figure $5 \mathrm{c}$ ).

\section{A high frequency of clinically relevant Aspergillus-associated disease occurs in bronchiectasis}

Prior work from our group and others highlights the association of Aspergillus-associated disease (aspergillosis) with adverse clinical outcomes across a range of chronic respiratory diseases [7, 13, 22]. Given the high frequencies of Aspergillus detected, we next evaluated the occurrence of aspergillosis and its clinical relevance in bronchiectasis. To do this, we used a modified immunological classification system developed for CF (supplementary table E4) [14]. In addition to the criteria described by BAXTER et al. [14], our modified version includes incorporation of potential A. terreus-associated disease (by inclusion of A. terreus specific IgE and qPCR) along with other minor modifications detailed in supplementary table E4. Of note, although total IgE was measured, it was not used in our classification of patients because it has been described that ABPA may occur at IgE concentrations below the described cut-offs in the established 

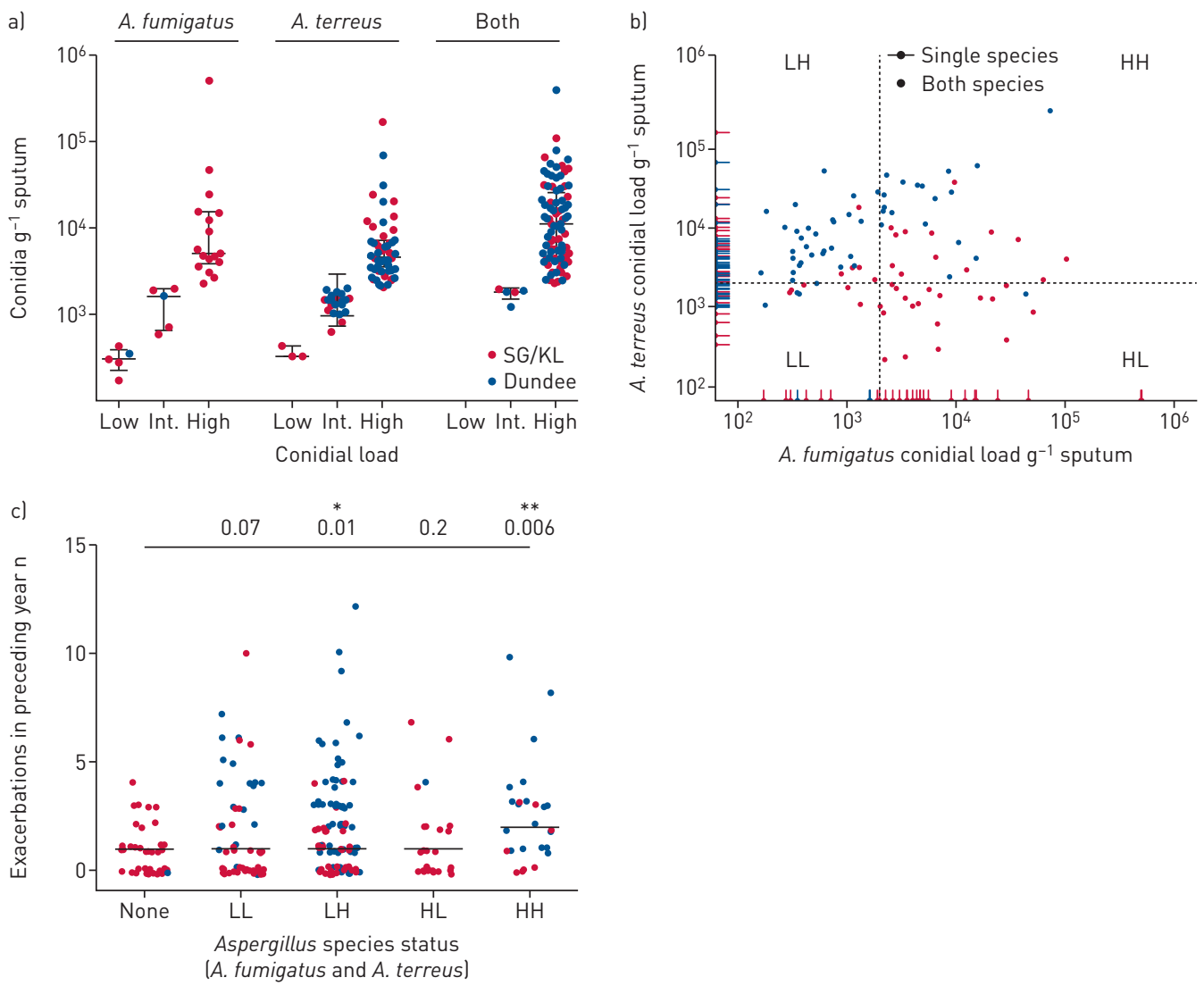

FIGURE 5 Quantification of airway Aspergillus conidial burden and its association with exacerbations in bronchiectasis ( $\mathrm{n}=238$ ). SG/KL: Singapore/Kuala Lumpur. a) Conidial burden per gram of sputum was quantified for $A$. fumigatus and $A$. terreus, respectively, and classified according to load as low (<500 conidia. ${ }^{-1}$ sputum), intermediate ("Int."; $500-2000$ conidia $\cdot g^{-1}$ sputum) or high (>2000 conidia $\cdot \mathrm{g}^{-1}$ sputum). Conidial load categories are illustrated according to fungal airway status as $A$. fumigatus only, $A$. terreus only or the presence of both species. b) Scatter plot of $A$. fumigatus ( $x$-axis) and $A$. terreus ( $y$-axis) conidial load in stable bronchiectasis. Patients with single and both species are indicated. Patients with both species are classified by their relative $A$. fumigatus and A. terreus burdens into low burden of both ("LL"), low burden of $A$. fumigatus and high burden of $A$. terreus ("LH"), high burden of $A$. fumigatus and low burden of A. terreus ("HL"), and high burden of both ("HH"). Dotted lines indicate cut-off levels for a high conidial burden (>2000 conidia. $g^{-1}$ sputum). c) Exacerbations for the year preceding study recruitment in bronchiectasis patients with detectable conidial burdens of both A. fumigatus and $A$. terreus as classified as in (b). Median number of exacerbations per group is indicated and Benjamini-Hochberg adjusted $p$-values for all groups compared with "None" are shown. *: $p=0.01{ }^{* *}: p=0.006$.

criteria [23]. Patients were grouped into the following five categories: nondiseased (ND), Aspergillus-colonised (AC), Aspergillus-sensitised (AS), serological ABPA (sABPA) and suspected chronic pulmonary aspergillosis (sCPA). Significant numbers in the CAMEB cohort met criteria for an Aspergillus-associated disease state (ND 1.7\%, AC 3.4\%, AS 76.5\%, sABPA 18.1\% and sCPA 0.3\%) (figure 6). Measured total serum IgE, Aspergillus specific IgG and sputum galactomannan varied between patient groups (supplementary figure E1). Specific IgE against crude antigens of A. fumigatus and A. terreus, respectively, was highest in AS $\left(0.77\right.$ and $\left.0.57 \mathrm{kU} \cdot \mathrm{L}^{-1}\right)$ and sABPA $\left(0.92\right.$ and $\left.0.93 \mathrm{kU} \cdot \mathrm{L}^{-1}\right)(\mathrm{p}<0.001)$ (figure $6 \mathrm{a}$ and $\mathrm{b}$ ). TARC (thymus and activation regulated chemokine), previously identified as a marker of CF-ABPA, was also assessed [24]. We found TARC to be a poor indicator of AS and/or sABPA in bronchiectasis, with high false-negative rates in both groups (figure $6 \mathrm{c}$ ). Additionally, false positives were also observed in a small number of ND patients (figure $6 c$ ). Patients with sABPA exhibited significantly more exacerbations, poorer pulmonary function and the severest disease compared with other groups (figure $6 \mathrm{~d}$ and $\mathrm{f}$ ). AS was associated with greater exacerbations, while AC portended toward more exacerbations with preserved pulmonary function and less severe disease (figure $6 \mathrm{~d}$ and $\mathrm{f}$ ). The AC, AS and sABPA states therefore appear to exist along an increasing continuum of disease severity. Only a single patient met criteria for sCPA, precluding further analysis of this group. Aspergillus-associated disease in bronchiectasis is therefore frequent, variable and clinically relevant, including in patients appearing clinically stable (a characteristic inclusion criteria for our study). 

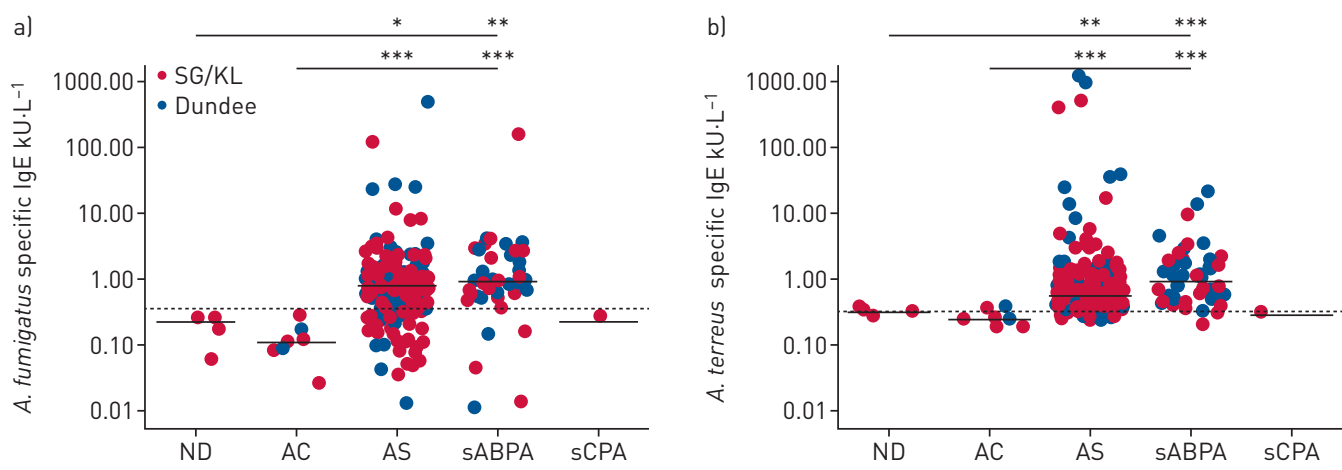

c)
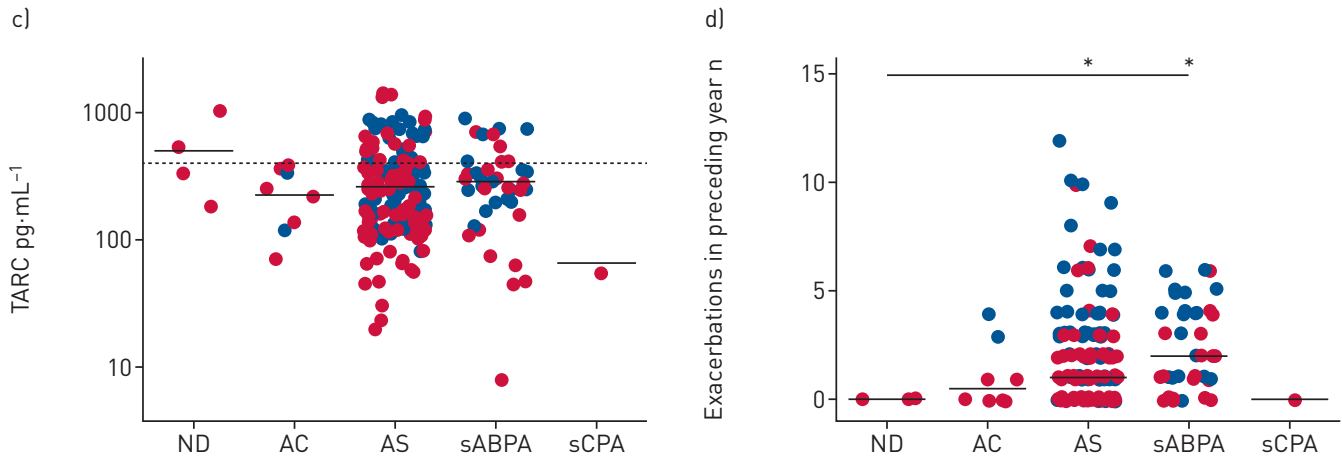

e)

f)
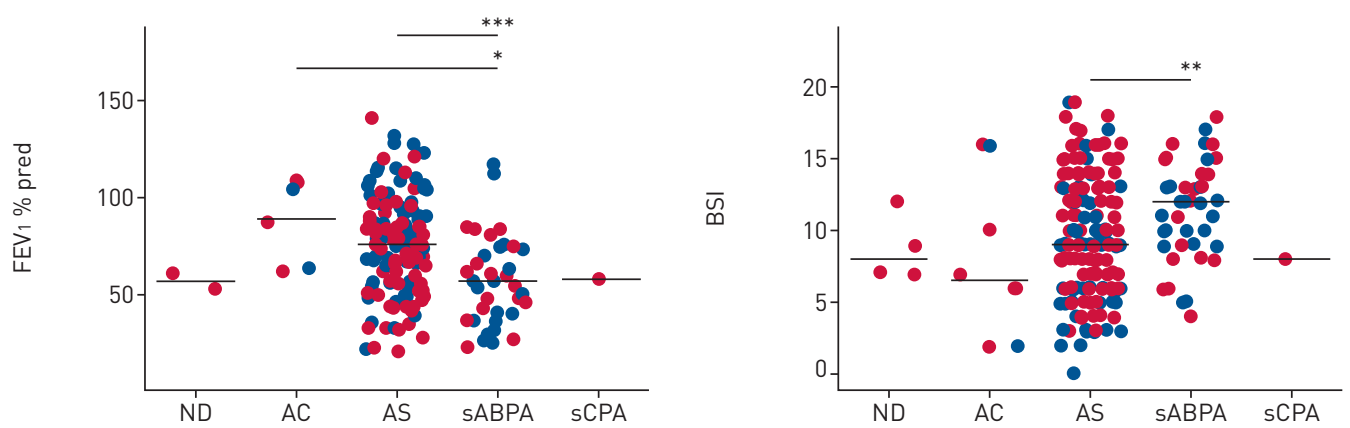

FIGURE 6 Immunological classification reveals high frequencies of aspergillosis in stable bronchiectasis and an association of serological allergic bronchopulmonary aspergillosis (sABPA) with greater exacerbations, poorer pulmonary function and more severe disease. SG/KL: Singapore/Kuala Lumpur; ND: nondiseased; AC: Aspergillus-colonised; AS: Aspergillus-sensitised; sCPA: suspected chronic pulmonary aspergillosis; TARC: thymus and activation regulated chemokine; FEV1: forced expiratory volume in $1 \mathrm{~s}$; BSI: Bronchiectasis Severity Index. a, b) Measured levels of specific IgE responses to a) $A$. fumigatus and b) $A$. terreus, where aspergillosis is classified immunologically as ND (n=4), AC (n=8), AS (n=182), sABPA (n=43) and sCPA (n=1) (supplementary table E4). c) TARC, a proposed ABPA marker in cystic fibrosis [24], was assessed according to the same classification. Dashed lines show respective cut-offs indicating a positive test for each marker (specific IgE $0.35 \mathrm{kU} \cdot \mathrm{L}^{-1}$; TARC $386 \mathrm{pg} \cdot \mathrm{mL}^{-1}$ ). d) Exacerbations for the year preceding study recruitment, e) pulmonary function (FEV1 \% pred) and f) disease severity (BSI) were assessed according to immunological aspergillosis class. Mean values are indicated except for exacerbations and BSI, where median values are shown. ${ }^{*}$ : $p<0.05 ;^{* *}: p<0.01 ;{ }^{* * *}: p<0.001$.

\section{Aspergillus-associated disease in bronchiectasis has distinct mycobiome profiles}

As high frequencies of Aspergillus-associated disease were observed in bronchiectasis based on our modified classification system, we next assessed for the presence of distinct taxonomic profiles that may associate with four of the five clinical states: ND, AC, AS and SABPA, excluding sCPA because this group contained only a single patient. Although unsupervised $\beta$ diversity analysis revealed three distinct mycobiome groups (supplementary figure E3), their association with clinical phenotype was not evident. We therefore adopted a supervised strategy comparing mycobiome profiles between our identified immunological classes. Each clinical state revealed mycobiome profiles characterised by a combination of varying fungal genera (figure 7). The ND group exhibited apparent high abundance of Phellinus, Magnusiomyces and Phlebia, with lower relative abundance of Saccharomyces, Aspergillus and Penicillium (figure 7a). 

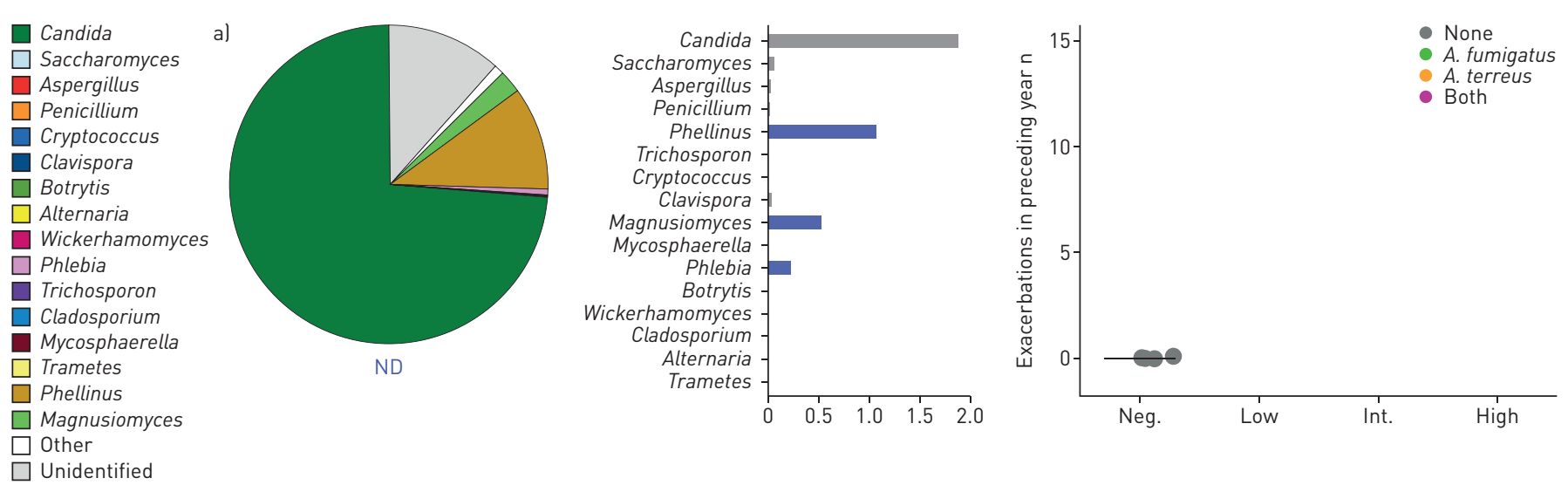

b)

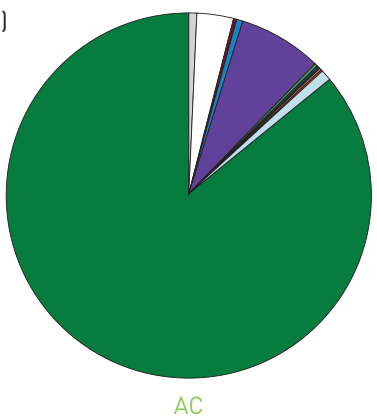

c)

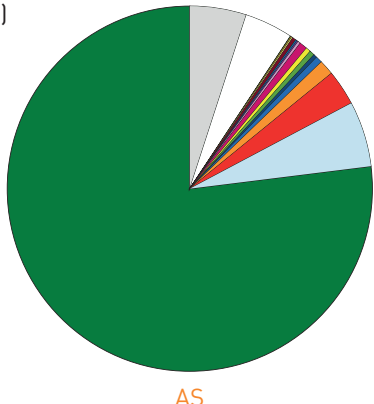

d)

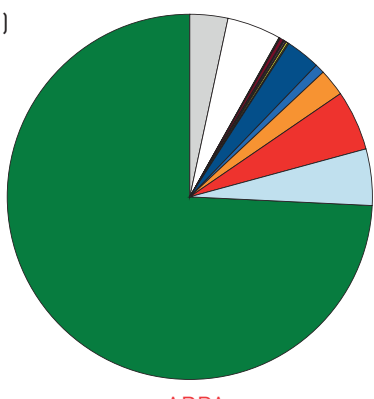

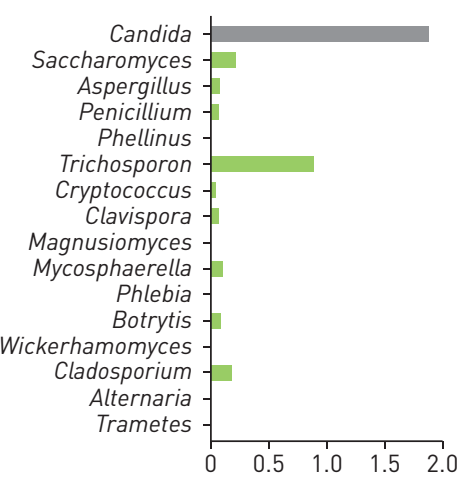

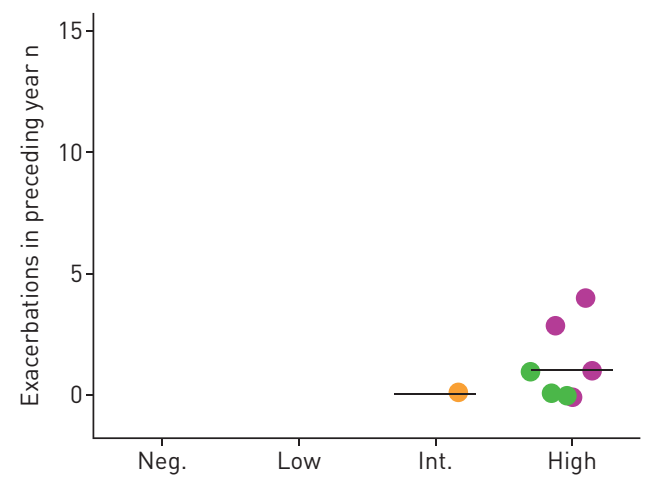

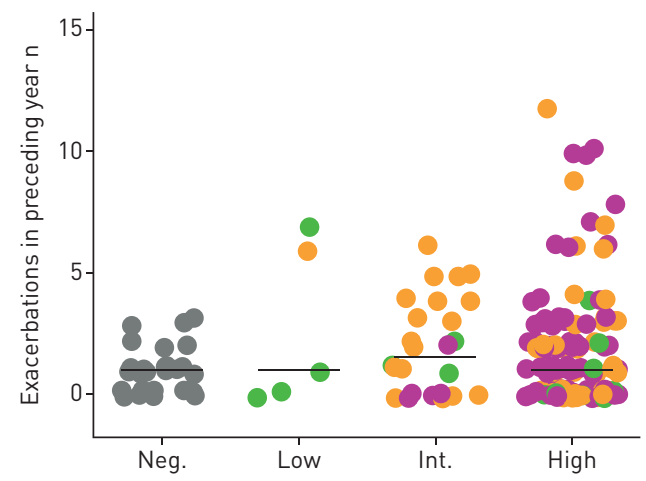
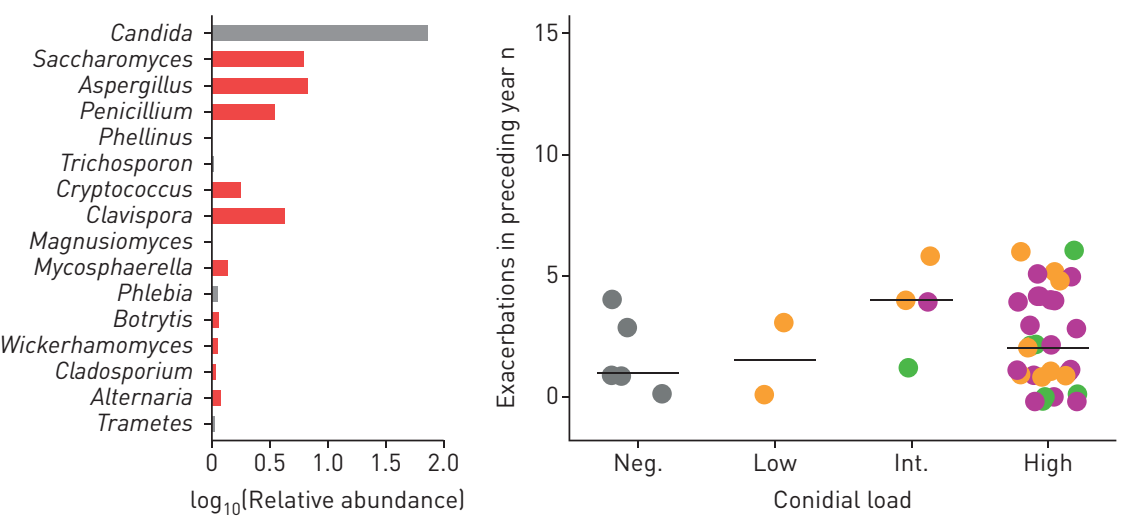
FIGURE 7 Pulmonary mycobiome profiles illustrate specific taxa-associated patterns according to immunological classification of aspergillosis in stable bronchiectasis. ND: nondiseased; AC: Aspergillus-colonised; AS: Aspergillus-sensitised; sABPA: serological allergic bronchopulmonary aspergillosis. a) ND ( $n=4)$, b) $A C(n=8)$, c) $A S(n=182)$ and d) sABPA ( $n=43)$ mycobiome profiles illustrate the different composition by relative abundance of reads classified to genus level, with the b) AC, c) AS and d) sABPA states exhibiting increased exacerbations and higher conidial load. Only a single patient had SCPA and therefore data is not shown. Mycobiome profiles are represented by pie charts with colour coding according to the taxonomic legend. Adjacent colour-coded log-scaled bar charts detail the observed taxa patterns in each immunological patient class (formal statistical assessment is provided in supplementary table E5). Exacerbations in the preceding year ( $y$-axis) are plotted against Aspergillus conidial load ( $x$-axis) and colour-coded according to quantitative PCR detection status of Aspergillus species into A. fumigatus alone, A. terreus alone, presence of both or none. Patients are further classified according to their individual conidial load as negative ("Neg."; no detected conidia. $g^{-1}$ sputum), low (<500 conidia.g ${ }^{-1}$ sputum), intermediate ("Int."; $500-2000$ conidia $\cdot g^{-1}$ sputum) or high ( $>2000$ conidia $\cdot g^{-1}$ sputum).

These latter three genera, however, increased in their relative abundance across a spectrum from ND to AC, AS and sABPA (figure 7 and supplementary table E5). The genera Cryptococcus and Clavispora followed similar and comparable spectra, with increasing relative abundance from AC, AS to sABPA. Mycosphaerella and Botrytis were present only in diseased states without a specific pattern (figure $7 \mathrm{~b}$ and d). Other characteristic fungal genera included Trichosporon and Cladosporium in the AC state, and Wickerhamomyces and Alternaria in the AS and sABPA states (figure 7b). Importantly, the relative abundance of Aspergillus detected from mycobiome profiles was in agreement with our qPCR-based quantification of conidial burden for A. fumigatus and A. terreus across all disease categories, with greater exacerbations noted in AS and SABPA (figure 7). Mycobiome profiles unique to each Aspergillus-associated disease state are therefore potentially useful for future bronchiectasis studies that focus on the diagnosis and endo-phenotyping of fungal disease.

\section{Discussion}

We describe the first, culture-independent analysis of the pulmonary mycobiome performed to date in bronchiectasis and, to the best of our knowledge, the first such respiratory study to meticulously match geographically distinct patient cohorts allowing us to assess potential regional variation in the mycobiome. By applying high-throughput 18S-28S ITS sequencing and other molecular techniques, we delineated the mycobiome constituents and their associated clinically relevant states. Our analysis identified Aspergillus as a major fungal genus in bronchiectasis, similar to that described in other chronic respiratory diseases $[6,12]$. An interesting observation was the prevalence of specific Aspergillus species characterising our Singapore/ Kuala Lumpur and Dundee cohorts, with A. fumigatus and A. terreus most frequently detected within each respective group, the latter associated to exacerbations. Further patient stratification into groups including AC, AS and sABPA states revealed high occurrences of clinically significant disease, even in "stable" patients, and the existence of distinct mycobiome profiles of discriminant taxa for each group.

Changes to regional lung growth conditions create a conducive environment for colonisation and infection by pathogenic microbes [2]. Given the gross anatomical distortion characteristic of bronchiectasis, it is therefore unsurprising that microbial differences are observed when compared with nondiseased states. The chronic bronchial insult coupled to anatomical distortion, compromised mucociliary clearance, sustained neutrophilic response and recurrent infection all favour the growth of pathogenic fungi and their ensuing clinical consequences in bronchiectasis $[6,25]$.

As observed in this and other studies, the airway mycobiome is dominated by Ascomycota [26]. Ascomycota-dominant profiles are characteristic of bronchiectasis, although an important minority have high airway Basidiomycota. Basidiospores are environmentally more abundant and smaller than ascospores, explaining their airway presence [27]. Compared with Ascomycota, which includes the established respiratory pathogens Aspergillus and Candida, the specific role of Basidiomycota in bronchiectasis is unclear. Notably, pathogenic Basidiomycota including Cryptococcus, Schizophyllum, Phellinus, Ceriporia and Trichosporon were all detected in our work [28]. Cryptococcus is significantly elevated in bronchiectasis, an observation previously documented that now warrants further investigation [29]. The existence of pulmonary Basidiomycota might reflect dynamic trafficking of "rare biosphere" fungi ("immigration and elimination"), suggestive of a healthier respiratory state rather than the sustained fungal outgrowth and unfavourable environment associated with Ascomycota $[2,5]$. The cross-sectional design of our study, however, precluded longitudinal assessment of the stability and transiency of the mycobiome over time, a key consideration for future work.

Bronchiectasis-associated fungal genera include Aspergillus, Issatchenkia, Wickerhamomyces and Simplicillium. Where dedicated species-specific qPCR for Aspergillus was performed, only A. fumigatus and $A$. terreus were identifiable in bronchiectasis. Interestingly, A. fumigatus had a preponderance within the Asian cohorts from Singapore/Kuala Lumpur, while A. terreus exhibited higher prevalence and burden in patients from Dundee. Even in patients with both species, geographic origin dictated which proportionally dominated. Our patients were stringently matched for age, sex and disease severity in order 
to confront the heterogeneity that plagues bronchiectasis research and allowed us to better assess potential geographic variation. Studies of additional cohorts from Europe and Asia will be required to determine if these are truly geographical differences or reflect differences in patient selection or referral patterns at the participating sites. These geographic phenotypes were accompanied by differences in lung mycobiome profiles: Penicillium, Clavispora and Cryptococcus had markedly higher frequency in patients from Dundee, while Basidiomycota genera Phlebia, Trichosporon, Ceriporia, Phellinus, Schizophyllum, Psathyrella and Peniophora were exclusive to patients from Singapore/Kuala Lumpur who exhibited higher overall Basidiomycota. Our identified differences may reflect a myriad of regionally variable factors such as contrasting atmospheric conditions between temperate and tropical climates including temperature and humidity, each documented to affect fungi [30,31]. Interestingly, our identified geographic variation of A. terreus predominance in Dundee occurred despite low reported UK prevalence rates of this fungus, suggestive of enrichment in the bronchiectasis population [32]. CF-related bronchiectasis also associates with $A$. terreus and an unidentified "environmental exposure" of likely relevance in our Dundee cohort is ascribed to this species [33, 34]. Other key geographic factors to consider include dietary preferences, genetics, air quality and/or lifestyle, all influences on acquisition and persistence of microbes including fungi [35]. Interestingly, the marked body mass index difference between our "matched" cohorts raises questions about potential differences in gut microbiomes that in turn may associate with the airway mycobiome given the emerging lung-gut axis relationship, an area of increasing relevance to respiratory health [36]. Other confounders to consider include regional differences in pharmacological prescribing and their consequent implications for the mycobiome. These are important considerations given differences between our Dundee and Singapore/Kuala Lumpur cohorts in terms of inhaled corticosteroids, long-term prophylactic antibiotics (greater in Dundee) and mucolytic use (greater in Singapore/Kuala Lumpur) (table 1), all features of potential influence on mycobiome composition that warrant further study. While our findings could be peculiar to Dundee, Singapore and Malaysia, generalisability is supported by data showing that Dundee patients are similar and data generated in Scotland has been generalisable across more than 10 European cohorts in recent analyses [37, 38].

Our group and others have previously investigated the role of Aspergillus-associated disease in CF-related bronchiectasis, although focused work in non-CF bronchiectasis is lacking [7, 35, 39-41]. Importantly, however, EvERAERTs et al. [22] assessed the occurrence of A. fumigatus sensitisation in COPD and, when detectable, associated it with a high risk for bronchiectasis. These data support the role for Aspergillus as a bronchiectasis pathogen either as a cause or consequence. Our data further corroborate such associations with high observable specific IgE levels to A. fumigatus and A. terreus. Using a modified immunological classification, adapted from CF, we describe high rates of AS and SABPA disease states in the CAMEB cohort, outlining its clinical relevance even in patients appearing clinically stable [14]. TARC, a proposed marker of CF-ABPA, was found to have poor sensitivity and specificity when applied to the CAMEB cohort, illustrating difficulties in translating findings from CF to non-CF bronchiectasis [24]. Each of our classified clinical states is accompanied by a distinct mycobiome pattern suggestive of mechanistic links necessitating future studies.

A potential role for A. terreus in bronchiectasis is a novel finding of this work. When compared with A. fumigatus, A. terreus is less studied. It is, however, noted for colonising the CF lung, is capable of causing ABPA and associates with opportunistic infections [42-44]. Its resistance to polyene antifungals confers survival advantages in the host compared with A. fumigatus [45, 46]. Although A. terreus conidia are rapidly phagocytosed through their recognition by dectin-1 and mannose receptors, they exhibit increased macrophage survival compared with A. fumigatus [46]. Rapid germination of A. fumigatus within the macrophage comes at the cost of its increased vulnerability to host immunity. In contrast, $A$. terreus germinates at lower rates favouring its persistence. Our work highlights high proportions of bronchiectasis patients with both A. fumigatus and A. terreus. Moreover, such patients demonstrate greater exacerbations, suggestive that in combination these fungi have important clinical effects on distinct host pathways that worsen disease. As a consequence, assessment of fungal airway conidial burden is important in bronchiectasis.

While our work is novel, it does have limitations including its cross-sectional design. This is a consequence of our attempt to robustly match Asian and European cohorts within the study to address disease heterogeneity, a key issue in bronchiectasis research. In addition, we must consider the "generalisability" of our three participating countries in comparison to the wider Asian and European subcontinents, including the potential variation in aetiology for exacerbations, their management including bronchiectasis treatments, and geographic differences in climate, temperature and air quality. Epidemiological fungal studies have in fact illustrated species differences between countries even within the same subcontinent [32]. Furthermore, we included only a small group of nondiseased (healthy) individuals (all from Singapore) that precluded us from defining a "healthy" mycobiome clearly, which was not the primary aim of this work. Future studies, however, should focus on assessing the nondiseased lung mycobiome and include individuals from more than a single region to assess potential geographic 
variation. In addition, targeted amplicon sequencing, even through our parallel shotgun sequencing of ITS1 and ITS2, has lower resolution compared with the more costly and analytically challenging whole-genome shotgun metagenomics approach that provides superior speciation and functional annotation. We focused on Aspergillus as the predominant bronchiectasis-associated fungal pathogen; however, our mycobiome analysis suggests that other fungal genera may also have roles. Finally, when the CAMEB cohort was conceived, a decision was made to match across Singapore/Kuala Lumpur and Dundee based on age, sex and disease severity. This matching on total BSI rather than its specific components, including exacerbations, radiology or microbiology, limits our ability to perform more specific analyses. Our cohort enrolled predominantly severe patients with bronchiectasis with a relatively low number of patients with mild disease (BSI 0-4).

In conclusion, we have performed the first fungal profiling study in bronchiectasis to date using two "matched" cohorts across three countries. We illustrate high levels of airway fungi and key differences between cohorts. These data suggest that the role for fungi, and specifically Aspergillus, in bronchiectasis is significant and routine screening for Aspergillus-associated diseases should be considered for inclusion in future bronchiectasis guidelines. Screening may include skin testing, Aspergillus-associated specific IgE and Aspergillus specific IgG with potential follow-up immunological monitoring for those clinically affected. Our identified mycobiome profiles that relate to clinical disease should also be elucidated in future work.

Conflict of interest: F.T. Chew reports personal fees from Sime Darby Technology Center, Olam International and First Resources Ltd, outside the submitted work. J.D. Chalmers reports grants from AstraZeneca, grants and personal fees from GlaxoSmithKline, Boehringer Ingelheim, Pfizer, Bayer Healthcare and Grifols, and personal fees from Napp, outside the submitted work.

Support statement: This research is supported by the Singapore Ministry of Health's National Medical Research Council under its Transition Award (NMRC/TA/0048/2016) (S.H. Chotirmall) and the Changi General Hospital Research Grant (CHF2016.03-P) (T.B. Low). The work performed at the National University of Singapore was supported by the Singapore Ministry of Education Academic Research Fund, the Singapore Immunology Network and the National Medical Research Council: N-154-000-038-001, R-154-000-404-112, R-154-000-553-112, R-154-000-565-112, R-154-000-630-112, R-154-000-A08-592, R-154-000-A27-597, SIgN-06-006, SIgN-08-020 and NMRC/1150/2008 (F.T. Chew). J.D. Chalmers is supported by the GSK/British Lung Foundation Chair of Respiratory Research. Funding information for this article has been deposited with the Crossref Funder Registry.

\section{References}

1 Polverino E, Goeminne PC, McDonnell MJ, et al. European Respiratory Society guidelines for the management of adult bronchiectasis. Eur Respir J 2017; 50: 1700629.

2 Dickson RP, Erb-Downward JR, Martinez FJ, et al. The microbiome and the respiratory tract. Annu Rev Physiol 2016; 78: 481-504

3 Rogers GB, Zain NM, Bruce KD, et al. A novel microbiota stratification system predicts future exacerbations in bronchiectasis. Ann Am Thorac Soc 2014; 11: 496-503.

4 Cox MJ, Turek EM, Hennessy C, et al. Longitudinal assessment of sputum microbiome by sequencing of the 16S rRNA gene in non-cystic fibrosis bronchiectasis patients. PLoS One 2017; 12: e0170622.

5 Huffnagle GB, Noverr MC. The emerging world of the fungal microbiome. Trends Microbiol 2013; 21: 334-341.

6 Chotirmall SH, Martin-Gomez MT. Aspergillus species in bronchiectasis: challenges in the cystic fibrosis and non-cystic fibrosis airways. Mycopathologia 2018; 183: 45-59.

7 Coughlan CA, Chotirmall SH, Renwick J, et al. The effect of Aspergillus fumigatus infection on vitamin D receptor expression in cystic fibrosis. Am J Respir Crit Care Med 2012; 186: 999-1007.

8 Chotirmall SH, O’Donoghue E, Bennett $\mathrm{K}$, et al. Sputum Candida albicans presages $\mathrm{FEV}_{1}$ decline and hospital-treated exacerbations in cystic fibrosis. Chest 2010; 138: 1186-1195.

9 McMahon MA, Chotirmall SH, McCullagh B, et al. Radiological abnormalities associated with Aspergillus colonization in a cystic fibrosis population. Eur J Radiol 2012; 81: e197-e202.

10 Chotirmall SH, McElvaney NG. Fungi in the cystic fibrosis lung: bystanders or pathogens? Int J Biochem Cell Biol 2014; 52: 161-173.

11 Chotirmall SH, Al-Alawi M, Mirkovic B, et al. Aspergillus-associated airway disease, inflammation, and the innate immune response. Biomed Res Int 2013; 2013: 723129.

12 Yii AC, Koh MS, Lapperre TS, et al. The emergence of Aspergillus species in chronic respiratory disease. Front Biosci 2017; 9: 127-138.

13 Goh KJ, Yii ACA, Lapperre TS, et al. Sensitization to Aspergillus species is associated with frequent exacerbations in severe asthma. J Asthma Allergy 2017; 10: 131-140.

14 Baxter CG, Dunn G, Jones AM, et al. Novel immunologic classification of aspergillosis in adult cystic fibrosis. J Allergy Clin Immunol 2013; 132: 560-566.

15 Leung JM, Tiew PY, Mac Aogain M, et al. The role of acute and chronic respiratory colonization and infections in the pathogenesis of COPD. Respirology 2017; 22: 634-650.

16 Lonni S, Chalmers JD, Goeminne PC, et al. Etiology of non-cystic fibrosis bronchiectasis in adults and its correlation to disease severity. Ann Am Thorac Soc 2015; 12: 1764-1770.

17 Aliberti S, Masefield S, Polverino E, et al. Research priorities in bronchiectasis: a consensus statement from the EMBARC Clinical Research Collaboration. Eur Respir J 2016; 48: 632-647.

18 Hill AT, Haworth CS, Aliberti S, et al. Pulmonary exacerbation in adults with bronchiectasis: a consensus definition for clinical research. Eur Respir J 2017; 49: 1700051. 
19 Ong SH, Kukkillaya VU, Wilm A, et al. Species identification and profiling of complex microbial communities using shotgun Illumina sequencing of 16S rRNA amplicon sequences. PLoS One 2013; 8: e60811.

20 White JR, Nagarajan N, Pop M. Statistical methods for detecting differentially abundant features in clinical metagenomic samples. PLoS Comput Biol 2009; 5: e1000352.

21 Walsh TJ, Wissel MC, Grantham KJ, et al. Molecular detection and species-specific identification of medically important Aspergillus species by real-time PCR in experimental invasive pulmonary aspergillosis. J Clin Microbiol 2011; 49: 4150-4157.

22 Everaerts S, Lagrou K, Dubbeldam A, et al. Sensitization to Aspergillus fumigatus as a risk factor for bronchiectasis in COPD. Int J Chron Obstruct Pulmon Dis 2017; 12: 2629-2638.

23 Agarwal R, Chakrabarti A, Shah A, et al. Allergic bronchopulmonary aspergillosis: review of literature and proposal of new diagnostic and classification criteria. Clin Exp Allergy 2013; 43: 850-873.

24 Hartl D, Latzin P, Zissel G, et al. Chemokines indicate allergic bronchopulmonary aspergillosis in patients with cystic fibrosis. Am I Respir Crit Care Med 2006; 173: 1370-1376.

25 Chalmers JD, Hill AT. Mechanisms of immune dysfunction and bacterial persistence in non-cystic fibrosis bronchiectasis. Mol Immunol 2013; 55: 27-34.

26 Ghannoum MA, Jurevic RJ, Mukherjee PK, et al. Characterization of the oral fungal microbiome (mycobiome) in healthy individuals. PLoS Pathog 2010; 6: e1000713.

27 Yamamoto N, Bibby K, Qian J, et al. Particle-size distributions and seasonal diversity of allergenic and pathogenic fungi in outdoor air. ISME J 2012; 6: 1801-1811.

28 Chowdhary A, Kathuria S, Agarwal K, et al. Recognizing filamentous basidiomycetes as agents of human disease: a review. Med Mycol 2014; 52: 782-797.

29 Li SS, Mody CH. Cryptococcus. Proc Am Thorac Soc 2010; 7: 186-196.

30 Lim SH, Chew FT, Binti Mohd Dali SD, et al. Outdoor airborne fungal spores in Singapore. Grana 1998; 37: $246-252$

31 O'Gorman CM, Fuller HT. Prevalence of culturable airborne spores of selected allergenic and pathogenic fungi in outdoor air. Atmos Environ 2008; 42: 4355-4368.

32 Risslegger B, Zoran T, Lackner M, et al. A prospective international Aspergillus terreus survey: an EFISG, ISHAM and ECMM joint study. Clin Microbiol Infect 2017; 23: 776.e1-776.e5.

33 Lackner M, Coassin S, Haun M, et al. Geographically predominant genotypes of Aspergillus terreus species complex in Austria: a microsatellite typing study. Clin Microbiol Infect 2016; 22: 270-276.

34 Rougeron A, Giraud S, Razafimandimby B, et al. Different colonization patterns of Aspergillus terreus in patients with cystic fibrosis. Clin Microbiol Infect 2014; 20: 327-333.

35 Chotirmall SH, Gellatly SL, Budden KF, et al. Microbiomes in respiratory health and disease: an Asia-Pacific perspective. Respirology 2017; 22: 240-250.

36 Budden KF, Gellatly SL, Wood DL, et al. Emerging pathogenic links between microbiota and the gut-lung axis. Nat Rev Microbiol 2017; 15: 55-63.

37 McDonnell MJ, Aliberti S, Goeminne PC, et al. Multidimensional severity assessment in bronchiectasis: an analysis of seven European cohorts. Thorax 2016; 71: 1110-1118.

38 Chalmers JD, Aliberti S, Filonenko A, et al. Characterization of the "frequent exacerbator phenotype" in bronchiectasis. Am J Respir Crit Care Med 2018; 197: 1410-1420.

39 Hector A, Chotirmall SH, Lavelle GM, et al. Chitinase activation in patients with fungus-associated cystic fibrosis lung disease. J Allergy Clin Immunol 2016; 138: 1183-1189.

40 Mirkovic B, Lavelle GM, Azim AA, et al. The basophil surface marker CD203c identifies Aspergillus species sensitization in patients with cystic fibrosis. J Allergy Clin Immunol 2016; 137: 436-443.

41 Gernez Y, Walters J, Mirkovic B, et al. Blood basophil activation is a reliable biomarker of allergic bronchopulmonary aspergillosis in cystic fibrosis. Eur Respir J 2016; 47: 177-185.

42 Laham MN, Carpenter JL. Aspergillus terreus, a pathogen capable of causing infective endocarditis, pulmonary mycetoma, and allergic bronchopulmonary aspergillosis. Am Rev Respir Dis 1982; 125: 769-772.

43 Dunne K, Prior AR, Murphy K, et al. Emergence of persistent Aspergillus terreus colonisation in a child with cystic fibrosis. Med Mycol Case Rep 2015; 9: 26-30.

44 Lass-Florl C, Griff K, Mayr A, et al. Epidemiology and outcome of infections due to Aspergillus terreus: 10-year single centre experience. Br J Haematol 2005; 131: 201-207.

45 Walsh TJ, Petraitis V, Petraitiene R, et al. Experimental pulmonary aspergillosis due to Aspergillus terreus: pathogenesis and treatment of an emerging fungal pathogen resistant to amphotericin B. J Infect Dis 2003; 188: 305-319.

46 Slesiona S, Gressler M, Mihlan M, et al. Persistence versus escape: Aspergillus terreus and Aspergillus fumigatus employ different strategies during interactions with macrophages. PLoS One 2012; 7: e31223. 